\title{
Application of Raman Spectroscopy for Tracing the Status of Silica Fume in Cementitious Materials
}

\author{
Yanfei Yue and Yun Bai \\ Advanced and Innovative Materials (AIM) Group, Department of Civil, Environmental \\ and Geomatic Engineering, University College London, England \\ Jing Jing Wang \\ Centre for Research on Adaptive Nanostructures and Nanodevices, Trinity College Dublin, Ireland
}

\begin{abstract}
Silica fume (SF) is an important component for manufacturing high performance concrete (HPC), owing to its superb pozzolanic reactivity and physical filling effects. However, application of SF in concrete may cause potential hazards issues. Although using SF in slurry form can somehow reduce the potential biotoxicity, the long-term stability and status of the SF particles within cementitious materials is still uncertain. In the current study, attempts were made to use Raman spectroscopy as an innovative alternative technique for tracing and identifying the status of SF both in its original SF slurry and in a 6-month-old hydrated cement paste. Light-optical microscope was also used to examine the morphology of the SF particles in the aforementioned samples. The results showed that under Raman spectroscopy, the various components of the SF in slurry, such as amorphous silica, silicon crystal, and carbon, were clearly recognised. In addition, the SF agglomerates formed in the slurry were also detected. On the other hand, the chemical composition, status, and morphology of both SF and SF agglomerates in the 6 -month-old paste were also identified. The study reported in this paper indicates that Raman spectroscopy could be a potential technique for tracing the status of SF, so that the potential safety hazards of SF can be monitored.
\end{abstract}

\section{INTRODUCTION}

Silica fume (SF) is a nanomaterial consisting mainly of amorphous silica spheres (85-95\%) with average particle diameter of $100 \mathrm{~nm}$. It is a supplementary cementitious materials (SCMs) often used for manufacturing high performance concrete (HPC) with promising properties such as superb longterm durability and high strength (Siddique \& Iqbal Khan, 2011). The contribution of SF to the high performance of concrete is mainly attributed to the following two mechanisms: (i) chemical effect due to the pozzolanic reaction. As SF mainly consists of highly reactive amorphous silica, they can react with calcium hydroxide $(\mathrm{CH})$ (usually arising from the hydration of Portland cement) to form additional calcium silicate hydrate ( $\mathrm{CSH}$ ) - the most important binding and strength giving hydration product in concrete; (ii) physical filling effects. Because of its very small particle size, SF can fill in the various pores in the hardened cement matrix, and hence enhance the density and the strength of hardened cement paste. Both mechanisms can lead to better durability and higher strength. However, whilst SF has numerous advantages, it also has the common issue facing all nanomaterials, i.e., potential safety hazards to living systems (Napierska, Thomassen, Lison, Martens, \& Hoet, 2010). This is induced by the ultrafine particles of SF and thus could be easily absorbed through the skin, lungs, or digestive tract, causing health risks to the living systems. Therefore, nowadays, it is preferred to use SF slurry (i.e., SF in aqueous dispersions) with a typical solid content of 50 wt.\% in practical operations. Although this can somehow avoid the safety hazards incurred to the operators to certain extent and decrease the difficulty encountered from handling dry bulk powders, the potential safety hazards of the SF used in the slurry form still exists and the long-term stability of unhydrated/hydrated SF in concretes is still unknown. In particular, the following two aspects need to be clearly understood, so that SF slurry can be confidently used in concrete:

(i) The status of SF particles in original SF slurry. It is now well established that dry densified SF exists almost in the form of SF agglomerates, i.e., nanospheres linked together into chains or clusters, rather than isolated sphere (Diamond, Sahu, \& Thaulow, 2004). However, the status of SF particles in SF slurry is still unknown.

(ii) The status of SF within cementitious materials over time. In theory, the amorphous silica in the $\mathrm{SF}$ is very reactive and can react with calcium hydroxide $(\mathrm{CH})$ to form additional calcium silicate hydrate $(\mathrm{CSH})$, contributing to the strength 
development of cementitious materials. However, unhydrated SFs were observed in the long-term hydrated cementitious materials manufactured by densified SF, which could be attributed to the fact that the SF agglomerates may only break down partially in normal concrete mixing processes (Diamond et al., 2004). Consequently, concerns over the possible unhydrated SF agglomerates in SF slurries arise. In particular, the status and the stability of these unreacted nanoparticles are considered to be extremely important for the safety of the living systems exposed directly or indirectly to the concrete structures.

Therefore, it is of great importance to trace the status of original SF and SF within cementitious materials over different time scales. The information obtained will be invaluable for the identification of the interaction mechanisms between SF and cementitious materials as well as the long-term stability and activity of the silica agglomerate, enabling the possible identification of the safety issue of SF in cementitious materials in the future.

The current study was, thus, aimed at establishing a protocol of using Raman spectroscopy to identify the SF in original SF slurry and also trace the status of SF within cementitious materials at the nanoscale over different time scales. Light-optical microscope was simultaneously used to demonstrate the morphology of the various samples. Corresponding to the two issues aforementioned, the experiment work carried out was mainly focused at the following two aspects: (i) to identify the status, composition, and also morphology of SF particles in the original SF slurry with Raman spectroscopy and the results was then used as bench-mark for the subsequent analysis and (ii) to trace the status of SF in much matured cementitious paste. It was aimed here to identify the possible existence of unhydrated-SF agglomerates in a 6-month-old cement paste manufactured with SF slurry. All the information obtained are used to demonstrate the potential of Raman spectroscopy based techniques for assessing the nanosafety of SF in concrete structure.

\section{EXPERIMENTAL}

\subsection{SF slurry}

The as-received SF slurry, a dark-grey slurry supplied by Elkem with water content of $50 \%$, was used as the raw material in this study. Its chemical composition is shown in Table 1. To characterise the individual SF particles with Raman spectroscopy, diluted SF slurry was prepared at a slurry-to-water ratio of $1: 5$ by hand mixing in order to achieve a better dispersion of the SF particles before the Raman characterisation.
Table 1. Chemical composition (\%) of Portland cement and silica fume.

\begin{tabular}{lcccccccc}
\hline & $\mathrm{SiO}_{2}$ & $\mathrm{Al}_{2} \mathrm{O}_{3}$ & $\mathrm{Fe}_{2} \mathrm{O}_{3}$ & $\mathrm{CaO}$ & $\mathrm{MgO}$ & $\mathrm{K}_{2} \mathrm{O}$ & $\mathrm{Na}_{2} \mathrm{O}$ & $\mathrm{SO}_{3}$ \\
\hline $\mathrm{PC}$ & 23.00 & 6.15 & 2.95 & 61.30 & 1.80 & 0.68 & 0.22 & 2.50 \\
$\mathrm{SF}$ & 93.00 & 0.70 & 1.20 & 0.30 & 1.20 & 1.80 & 1.50 & 0.30 \\
\hline
\end{tabular}

\subsection{SF blended cement paste}

The Portland cement (PC) used in this study was CEM I (in accordance with BS EN 197-1:2011) supplied by QUINN and its chemical composition is given in Table 1 as well. The SF blended paste was prepared by replacing $8 \%$ of PC with SF (a typical level of SF used in practice) at a water/binder of 0.40 . Immediately after mixing, the paste was cast and sealed into disposable plastic centrifugal tubes to avoid loss of moisture. After $24 \mathrm{~h}$ initial curing in the tube, the specimens were removed from the tube and then covered with a water saturated hessian, which were then sealed in plastic sample bags and stored in a curing room at constant temperature of $20 \pm 1^{\circ} \mathrm{C}$ for around 6 months. The wet hessian was regularly checked and replaced once the moisture level was low. At the end of the sixth month, selected pastes were ground into the powder with fineness of $63 \mu \mathrm{m}$ and ready to be analysed.

\subsection{Raman spectroscopy combined with light-optical microscope}

Raman spectroscopy was applied for qualitative identification of the phases existing both in the SF and in the hardened cement pastes, whereas optical microscope was employed to characterise the dispersion of the silica nanoparticles and the morphology of various hydrated phases. Raman spectra were record at a controlled temperature of $20^{\circ} \mathrm{C}$ via a Renishaw micro-Raman spectroscopy equipped with a Charged Coupled Device (CCD) detector. The $488 \mathrm{~nm}(\mathrm{Ar}+)$ laser was used as excitation source. The laser beam was focused onto the samples through an Olympus $100 \times$ magnification microscope objective. The measured power at the sampling level was controlled at about $0.7 \mathrm{~mW}$. This low power level was purposely selected in order to avoid the overheating incurred from the dark grey SF samples under the laser interrogation. The Raman spectra were recorded with exposure time of $10 \mathrm{~s}$ and accumulations of 10 in order to improve the signal-to-noise ratio (SNR). Optical photograph was recorded with the objectives at different magnifications $(10 \times, 50 \times$, and $100 x)$ in order to acquire the appropriate resolution and features of the images. 


\section{RESULTS AND DISCUSSION}

\subsection{Characterisation of SF slurry with Raman} spectroscopy and light-optical microscope

To clearly identify the SF and any possible agglomeration of SF particles as well as to reveal the morphology of individual SF particles, the SF slurry was diluted at a water to SF slurry ratio of $5: 1$ which was then characterised under two optical objectives with different magnifications (i.e., 10x and 50x) together with the Raman spectroscopy analysis. The related results are shown in Figure 1 below.

As shown in Figure 1, under the magnification of $10 \times$, various dark clusters, which could be the silica agglomerates, can be observed floating in the diluted slurry. Under the magnification of $50 \times$ [Figure 1(c)], the silica agglomerates can be clearly identified consisting of spheres of individual SF particles. Under each magnification, Raman analysis was also carried out to identify the composition of the SF samples. As can be seen from Figures 1(b) and (d), SF comprises amorphous silica $\left(300-520 \mathrm{~cm}^{-1}\right)$, silicon crystals $\left(519 \mathrm{~cm}^{-1}\right)$ (Deschaines, Hodkiewicz, \& Herison, 2009), amorphous carbon (1360 and $1609 \mathrm{~cm}^{-1}$ ), and also uncertain impurities $\left(981 \mathrm{~cm}^{-1}\right)$. The above results from the diluted SF slurry would suggest that considerable amount of silica agglomerates exist in normal SF slurry. Therefore, it could be inferred that considerable amount of SF agglomerates could also exist in the hardened PC-SF pastes due to the difficulty in dispersing the silica particles in concentrated matrix. This could raise the concerns over the status of unreacted SF particles over time, especially in the long-term hydrated pastes, because any instability of these nanoparticles would cause potential nanosafety issues in reality. Some pilot studies were, hence, carried out in the current study to address the above issues and the related results are reported and discussed in Section 3.2.

\subsection{Characterisation of a 6-month SF blended paste with Raman spectroscopy and light-optical microscope}

To reveal the status of the SF in matured samples so that some useful information could be obtained to understand the possible situation of the SF in real structures, the SF blended cement paste was analysed with Raman spectroscopy after 6 months hydration. Again, the lightoptical spectroscopy was conducted to investigate the morphology, so that some typical features could be identified before carrying out the Raman analysis. As shown in Figure 2, an area with some typical features was selected. Locations similar to the feature of the typical SF agglomerate formed by the unhydrated SF particles (as presented in Figure 1) were also identified in this selected area. Four analysing points, i.e., A, B, C, and D, were then selected to carry out the Raman analysis.

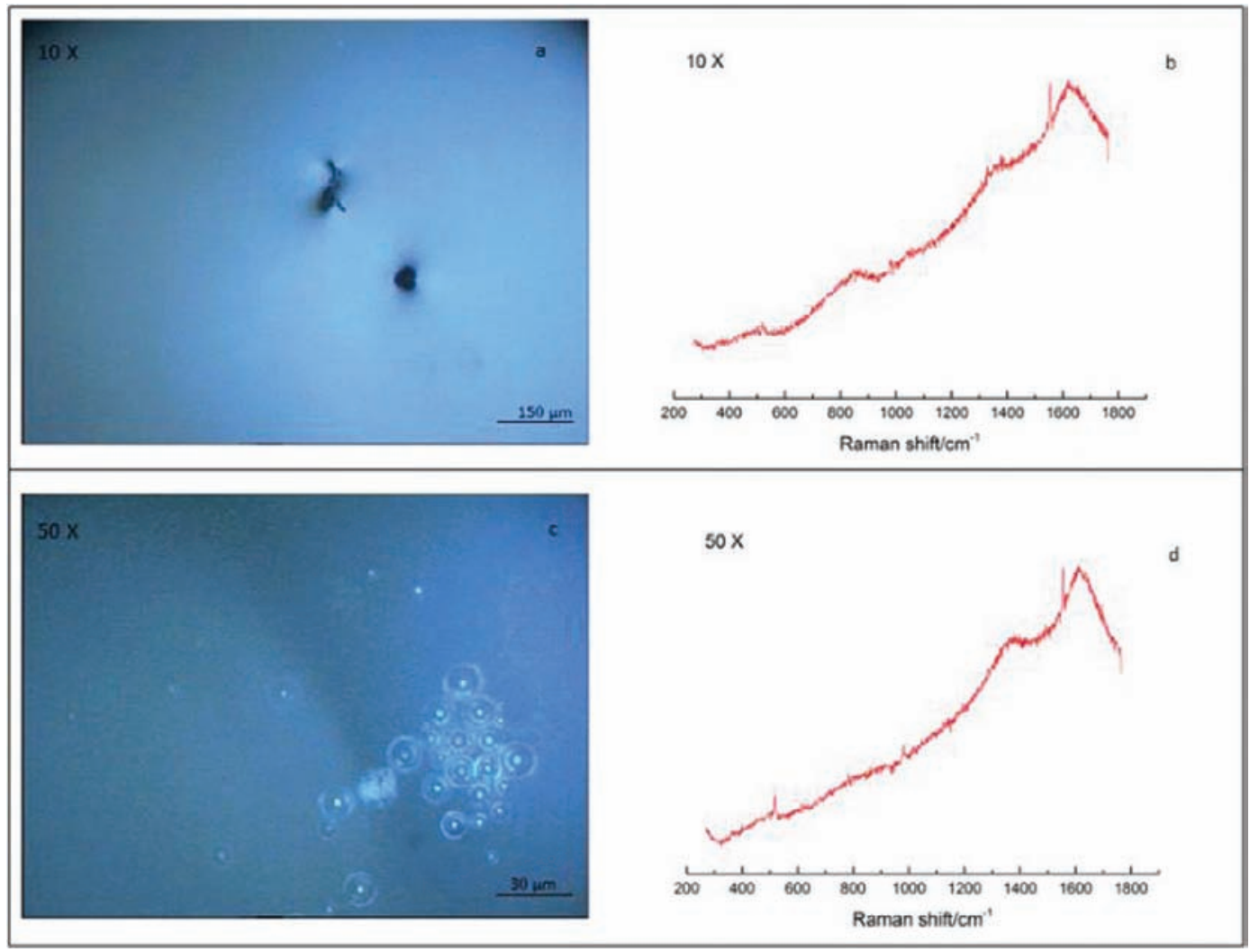

Figure 1. Light-optical micrograph and Raman spectra of diluted SF slurry under different magnifications. 


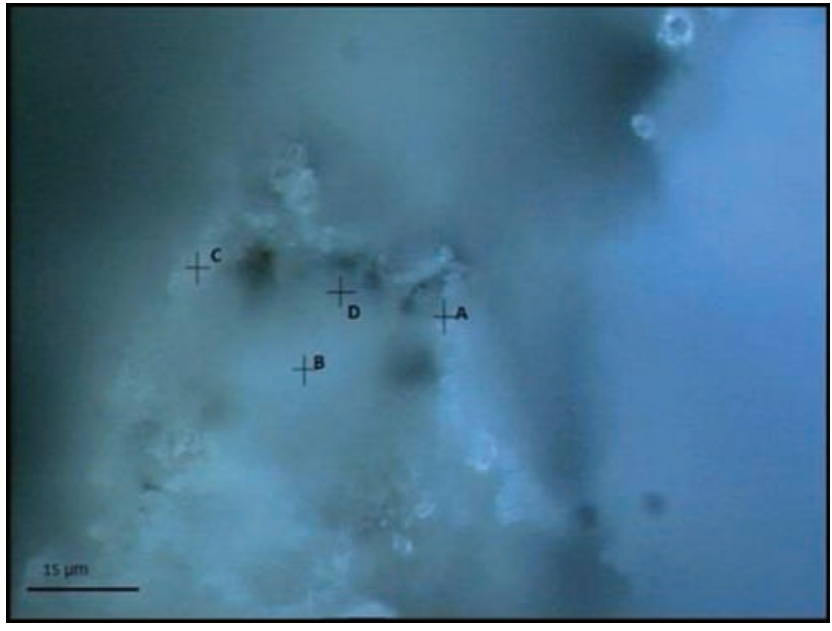

Figure 2. Light-optical micrograph showing the selected testing locations of the SF paste analysed after 6 months hydration (100x).

It can be seen from Figure 3, the Raman spectra were accompanied by strong fluorescence, together with the broad bands near 1380 and $1600 \mathrm{~cm}^{-1}$, which is the typical Raman features of carbon existed in the SF. The Raman features of the four analysing points are as follows:

(i) On the Raman spectrum of point $\mathrm{A}$, a weak band was identified at about $518 \mathrm{~cm}^{-1}$, which could be unhydrated SF. The peak located at $356 \mathrm{~cm}^{-1}$ could be attributed to the $\mathrm{Ca}-\mathrm{O}$ vibration of $\mathrm{Ca}(\mathrm{OH})_{2}$ formed during the hydration of PC. In addition, calcium silicate hydrate $(\mathrm{CSH})$ was also identified, as indicated by the three humps located at $600-710,820-900$, and $950-1020 \mathrm{~cm}^{-1}$, respectively. At the same time, there was a weak peak at $1084 \mathrm{~cm}^{-1}$, indicating the existence of carbonates;

(ii) For Point B, the sharp peaks at 1085 and $277 \mathrm{~cm}^{-1}$ could be assigned to the symmetric stretching of $\mathrm{CO}_{3}^{2-}$ and lattice vibration in the carbonates respectively. The $\mathrm{CSH}$ was again identified by the humps at $620-700,820-870$, and 940$1030 \mathrm{~cm}^{-1}$. Besides, the unhydrated amorphous silica was also recognised as evidenced by the hump around $400-530 \mathrm{~cm}^{-1}$, although it is very weak possibly due to its small quantify and the interference from the background fluorescence;

(iii) Point $C$ shows similar Raman pattern as that of Point $\mathrm{B}$, within which carbonates and $\mathrm{CSH}$ dominate the Raman spectra. It needs to be noticed that a peak at around $528 \mathrm{~cm}^{-1}$ was identified, which could be the unhydrated silicon crystals from SF;

(iv) Point $\mathrm{D}$ shows similar Raman bands as that of Point $\mathrm{A}$ with $\mathrm{CH} / \mathrm{CSH}$ were recognised. Additionally, there was a hump at $400-540 \mathrm{~cm}^{-1}$, which should be attributed to the unhydrated amorphous silica.

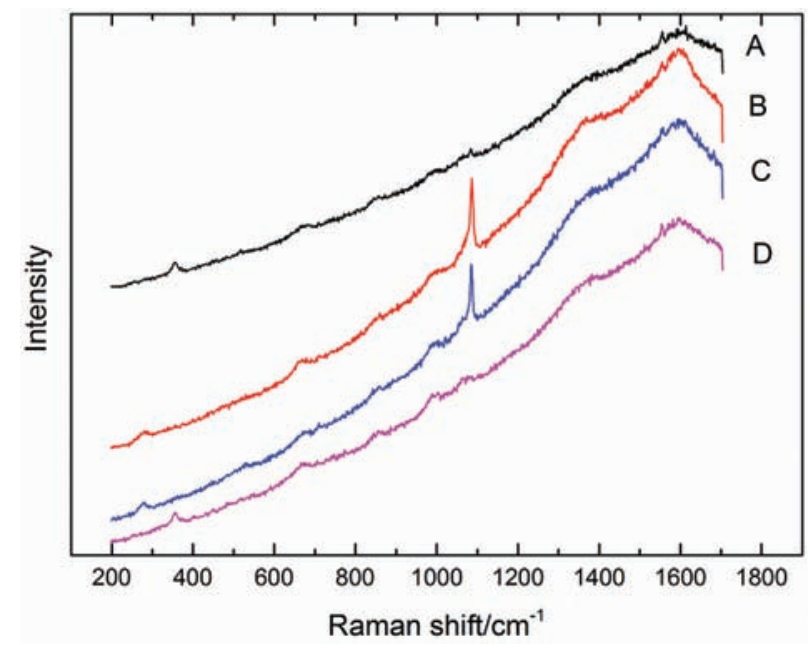

Figure 3. Raman spectra of the analysed points as shown in Figure 2.

As discussed, the spectra of the points $A$ and $D$ are very similar whilst those of the points $B$ and $C$ are similar. Taking into account of the optical micrograph in Figure 2, the following assumption and discussion have been deduced: the selected area could be a SF agglomerate covered by layers of $\mathrm{CH} / \mathrm{CSH}$ precipitates with inner core consisting of unhydrated SF particles. This is supported by the finding from the light-optical micrograph and Raman spectra which showed that the $\mathrm{CH}$ and $\mathrm{CSH}$ phases are identified at the outer area, i.e., points $A$ and $D$, whilst the unhydrated amorphous $\mathrm{SiO}_{2}$ is identified in the inner point $\mathrm{B}$. In addition, this deduction can be further confirmed by the literature which showed that the reactions between SF and $\mathrm{CH}$ will be retarded because of the formation of the $\mathrm{CSH}$ layer on the surface of SF agglomerate, which can prohibit the further reactions (Mitchell, Hinczak, \& Day, 1998).

\section{CONCLUSIONS}

Under Raman analysis with excitation laser of $488 \mathrm{~nm}$, various phases in original SF slurry have been successfully identified in this study, such as amorphous $\mathrm{SiO}_{2}\left(350-520 \mathrm{~cm}^{-1}\right)$, crystal silicon $\left(518 \mathrm{~cm}^{-1}\right)$, and amorphous carbon (1339 and $1608 \mathrm{~cm}^{-1}$ ). In addition, SF agglomerate in diluted SF slurry was also clearly observed by light-optical microscope and its chemical composition was identified by Raman spectroscopy. Furthermore, SF agglomerate was also identified in the 6-month hydrated SF blended paste, with a possible structure of analysis point being a cluster with the core composed of unhydrated SF particles and covered 
by a layer of $\mathrm{CSH}$ gel or $\mathrm{CH}$ crystal. These results indicate that there is a potential safety hazards to the living system due to the existence of unreacted SF particles in matured cementitious matrices.

\section{ACKNOWLEDGMENT}

This research was supported by the QNano Project http://www.qnano-ri.eu which is financed by the European Community Research Infrastructures under the FP7 Capacities Programme (Grant No. INFRA-2010-262163).

\section{REFERENCES}

Deschaines, T., Hodkiewicz, J., \& Herison, P. (2009). Characterization of amorphous and microcrystalline silicon using Raman spectroscopy. Spectroscopy Application Notebook, 24, 22.

Diamond, S., Sahu, S., \& Thaulow, N. (2004). Reaction products of densified silica fume agglomerates in concrete. Cement and Concrete Research, 34, 1625-1632.

Mitchell, D., Hinczak, I., \& Day, R. (1998). Interaction of silica fume with calcium hydroxide solutions and hydrated cement pastes. Cement and Concrete Research, 28, 1571-1584.

Napierska, D., Thomassen, L. C., Lison, D., Martens, J. A., \& Hoet, P. H. (2010). The nanosilica hazard: another variable entity. Particle and Fibre Toxicology, 7, 1.

Siddique, R., \& Iqbal Khan, M. (2011). Silica fume. Supplementary Cementing Materials Berlin, Heidelberg: Springer. 